\title{
Importance of false lumen thrombosis in type B aortic dissection prognosis
}

\author{
Santi Trimarchi, MD, PhD, ${ }^{a}$ Jip L. Tolenaar, MD, ${ }^{a}$ Frederik H. W. Jonker, MD, PhD, ${ }^{b}$ Brian Murray, MD, ${ }^{c}$ \\ Thomas T. Tsai, MD, ${ }^{\mathrm{d}}$ Kim A. Eagle, MD,${ }^{\mathrm{e}}$ Vincenzo Rampoldi, MD, ${ }^{\mathrm{a}}$ Hence J. M. Verhagen, MD, PhD, ${ }^{f}$ \\ Joost A. van Herwaarden, MD, PhD, ${ }^{g}$ Frans L. Moll, MD, PhD, ${ }^{g}$ Bart E. Muhs, MD, PhD, ${ }^{\text {h }}$ and \\ John A. Elefteriades, MD $^{\mathrm{c}}$
}

\begin{abstract}
Background: Partial thrombosis of the false lumen has been reported as a significant predictor of mortality during follow-up in patients with acute type B aortic dissection. The purpose of this study was to investigate the correlation of false lumen thrombosis and aortic expansion during follow-up in patients with acute type B aortic dissection.

Methods: All medically treated patients with acute type B aortic dissection observed in 4 cardiovascular referral centers between 1998 and 2011, with admission and follow-up computed tomography or magnetic resonance imaging scans, were included. Aortic diameters of the dissected aortas were measured at 4 levels on the baseline and follow-up scans, and annual growth rates were calculated. Univariate and multivariate regression analyses were used to investigate the effect of false lumen thrombosis on aortic growth rate.
\end{abstract}

Results: A total of 84 patients were included, of whom $40(47.6 \%)$ had a partially thrombosed false lumen, $7(8.3 \%)$ had a completely thrombosed false lumen, and $37(44.0 \%)$ had a patent false lumen. A total of 273 of the $336(81.3 \%)$ evaluated aortic levels were dissected segments. Overall, the mean aortic diameter increased significantly at all evaluated levels $(P<.001)$. Univariate analysis showed that annual aortic growth rates were significantly higher in those segments having a false lumen with partial thrombosis (mean, $4.25 \pm 10.2$ ) when compared with the patent group (mean, $2.10 \pm 5.56 ; P=.035$ ). In multivariate analysis, partial lumen thrombosis was an independent predictor of higher aortic growth (adjusted mean difference, $2.05 \mathrm{~mm} / \mathrm{year}$; 95\% confidence interval, $0.10-4.01 ; P=.040$ ).

Conclusions: In patients with acute type B aortic dissection, aortic segments with a partially thrombosed false lumen have a significantly higher annual aortic growth rate when compared with those presenting with patent or complete thrombosis of the false lumen. Therefore, patients with partial thrombosis require more intensive follow-up and may benefit from prophylactic intervention. (J Thorac Cardiovasc Surg 2013;145:S208-12)

The majority of patients affected by acute type B aortic dissection (ABAD), when uncomplicated, can be managed in the acute setting with medical therapy alone. Surgery or endovascular treatment is reserved for patients with

From the Thoracic Aortic Research Center, ${ }^{a}$ Policlinico San Donato IRCCS, Milan,

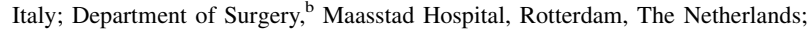
Section of Cardiac Surgery, ${ }^{c}$ Yale University School of Medicine, New Haven, Conn; Division of Cardiology \& Interventional Cardiology, ${ }^{\mathrm{d}}$ Denver VA Medical Center, Denver, Colo; Cardiovascular Center, ${ }^{\mathrm{e}}$ University of Michigan Health System, Ann Arbor, Mich; Department of Vascular Surgery, ${ }^{\mathrm{f}}$ Erasmus Medical Center, Rotterdam, The Netherlands; Department of Vascular Surgery, ${ }^{\mathrm{g}}$ University Medical Center Utrecht, Utrecht, The Netherlands; Section of Vascular Surgery, ${ }^{\mathrm{h}}$ Yale University School of Medicine, New Haven, Conn.

Disclosures: Drs Trimarchi, Tolenaar, Jonker, Murray, Tsai, Eagle, Rampoldi, Verhagen, van Herwaarden, Moll, Muhs, and Elefteriades have nothing to disclose with regard to commercial support.

Read at The American Association for Thoracic Surgery Aortic Symposium, New York, New York, April 26-27, 2012.

Received for publication April 24, 2012; revisions received Aug 7, 2012; accepted for publication Nov 20, 2012; available ahead of print Dec 20, 2012.

Address for reprints: Santi Trimarchi, MD, PhD, Thoracic Aortic Research Center, Policlinico San Donato IRCCS, University of Milano, Piazza Malan 2, 20097 San Donato Milanese MI, Italy (E-mail: santi.trimarchi@unimi.it).

0022-5223/\$36.00

Copyright (C) 2013 by The American Association for Thoracic Surgery

http://dx.doi.org/10.1016/j.jtcvs.2012.11.048 complications such as rupture, enlarging aneurysm, refractory hypertension or pain, retrograde dissection, or malperfusion syndromes. ${ }^{1}$ In-hospital outcomes are generally acceptable in patients with uncomplicated ABAD, with up to $90 \%$ of patients surviving to hospital discharge after receiving effective antihypertensive therapy. ${ }^{2,3}$ However, 1 in 4 patients with acute aortic dissection discharged from the hospital alive will die within 3 years, which exceeds the cumulative incidence of mortality in other diseases, such as coronary artery disease, moderate chronic obstructive pulmonary disease, and stage II colon cancer. ${ }^{3,4}$

Imaging studies suggest that complete thrombosis of the false lumen has beneficial prognostic value, whereas a patent false lumen predicts poor outcome ${ }^{5-9}$ Reports from the International Registry of Acute Aortic Dissection (IRAD) showed that a partially thrombosed false lumen is associated with increased surgical mortality, ${ }^{10}$ and among patients with $\mathrm{ABAD}$ who are discharged alive, those presenting with a partially thrombosed false lumen had increased mortality, after adjusting for age, gender, and type of in-hospital treatment. ${ }^{11}$ A hypothesis for this observation is that partial thrombosis of the false lumen may occlude 


\section{Abbreviations and Acronyms \\ $\mathrm{ABAD}=$ acute type $\mathrm{B}$ aortic dissection \\ IRAD = International Registry of Acute Aortic Dissection}

distal reentry tears ("sac formation"), resulting in higher diastolic pressure. ${ }^{12}$ This process may lead to an increased aortic wall tension and higher risk of aortic expansion, redissection, and rupture. The current study analyzes whether the presence of thrombosis in the false lumen in patients with $\mathrm{ABAD}$ is associated with increased aortic expansion.

\section{MATERIALS AND METHODS \\ Patient Selection}

Four cardiovascular referral centers participated in this project: Yale New Haven Hospital (New Haven, Conn), Erasmus University Medical Center (Rotterdam, The Netherlands), Policlinico San Donato IRCCS (Milan, Italy), and University Medical Center Utrecht (Utrecht, The Netherlands).

Patients with uncomplicated type B dissection who were admitted between 1998 and 2011 to these hospitals, with imaging studies obtained within 7 days of the diagnosis (baseline) and a second study (follow-up) obtained at least 1 month later, were analyzed. Patients who underwent an aortic intervention within 1 month after admission because of complications were excluded for analysis. Follow-up scans were obtained after 1 and 6 months of follow-up and yearly thereafter. The most recent imaging study was used for the analysis if multiple imaging studies were performed during follow-up.

\section{Imaging Analysis}

All imaging studies, computed tomography or magnetic resonance imaging, at admission and follow-up were selected for analysis and diameters of the affected aortas measured at the following levels: proximal descending aorta, defined as the level $2 \mathrm{~cm}$ below the origin of the left subclavian artery; distal descending aorta, defined as $20 \mathrm{~cm}$ below the origin of the left subclavian artery; visceral aorta, between the origin of the celiac trunk and superior mesenteric artery, and infra-renal aorta, measured below the origin of the most distal renal artery. The annual aortic growth rate was calculated at each affected level by dividing the difference in maximum aortic diameter in millimeters between the initial and final measurements by the time interval between the imaging examinations in years. In this manner, a total of 336 aortic levels were evaluated, of which 273 were dissected.

In addition, a core imaging laboratory of physicians with extensive experience and expertise in cross-sectional imaging assessed the flow dynamics of the false lumen as patent, partially thrombosed, or completely thrombosed. Patent false lumen was defined as the absence of thrombus in the false lumen, complete thrombosis was defined as the absence of contrast in the false lumen, and partial lumen thrombosis was defined as the presence of both thrombosis and contrast in the false lumen.

\section{Statistical Analysis}

Univariate linear regression analysis was used to analyze the association between the annual aortic growth of aortic segments and the age at presentation, gender, initial aortic diameter, and status of the false lumen classified as patent, partially, or completely thrombosed. All variables with a $P$ value less than .2 were entered in a multivariate regression model to calculate independent effects on the aortic growth rate. Statistical analysis was performed using the Statistical Package for the Social Sciences version
15.0 (SPSS Inc, Chicago, Ill). Data are shown as mean \pm standard deviation or median and interquartile range.

\section{RESULTS}

Eighty-four patients were included, of whom $40(47.6 \%)$ had a partially thrombosed false lumen, $7(8.3 \%)$ had a completely thrombosed false lumen, and $37(44.0 \%)$ had a patent false lumen. During follow-up, false lumen status changed from patent to partial thrombosis in 8 patients, partial thrombosis to patent false lumen in 2 patients, and partial to complete thrombosis in 4 patients. The mean aortic diameter at baseline was significantly larger in the partial lumen thrombosis group $(38.0 \pm 12.0)$ compared with the complete thrombosis group $(35.2 \pm 8.4)$ and the patent false lumen group $(32.0 \pm 6.9, P<.001)$. The median length of follow-up was 19.5 months (interquartile range, 43.7; range, $1-149.5$ months). A total of 273 of the $336(81.3 \%)$ evaluated aortic levels were dissected. Overall, aortic expansion was observed at 102 of the 125 segments $(81.6 \%)$ with a patent false lumen, at 98 of the 125 segments $(78.4 \%)$ in case of partial false lumen thrombosis, and at 15 of the 23 levels $(65.2 \%)$ in complete thrombosis. Overall, the mean diameter of the aortic segments significantly increased from $34.3 \pm 9.9$ at baseline to $37.2 \pm 12.4$ at latest follow-up $(P<.001)$. The increase was significant at all evaluated levels during follow-up (Figure 1). The mean aortic expansion rate was $3.0 \pm 8.1 \mathrm{~mm} /$ year. Mean annual aortic growth rates were $4.3 \pm 10.2 \mathrm{~mm} /$ year in those with a partial thrombosis, $2.1 \pm 5.6 \mathrm{~mm} /$ year in those with a patent false lumen, and $1.5 \pm 5.6 \mathrm{~mm} / \mathrm{year}$ in those with a completely thrombosed false lumen (Table 1).

Univariate analysis showed that age, male gender, and partial thrombosis of the false lumen (vs patent false lumen) had a significant effect on the annual aortic growth rate $(P<.05$ in all). Annual aortic expansion was significantly larger in patients with a partially thrombosed false lumen compared with a patent false lumen $(P=.035$, Table 2$)$. There was no significant difference between the annual aortic growth rates of dissections in patients with a partially thrombosed false lumen and patients with a completely thrombosed false lumen $(P=.745)$.

The results of the multivariate linear regression analysis are presented in Table 3. Those who were older at presentation had a $0.17-\mathrm{mm}$ per year decrease in aortic growth with every year increase in age $(95 \%$ confidence interval, -0.026 to $-0.08 ; P<.001)$. Partial lumen thrombosis resulted in a $2.05 \mathrm{~mm} /$ year increase in aortic growth compared with patients with a patent false lumen $(95 \%$ confidence interval, $0.10-4.01 ; P=.040$ ).

\section{DISCUSSION}

Aortic expansion during follow-up will develop in a substantial number of medically treated patients with $\mathrm{ABAD}$, and 1 in 4 patients who are discharged alive from the 

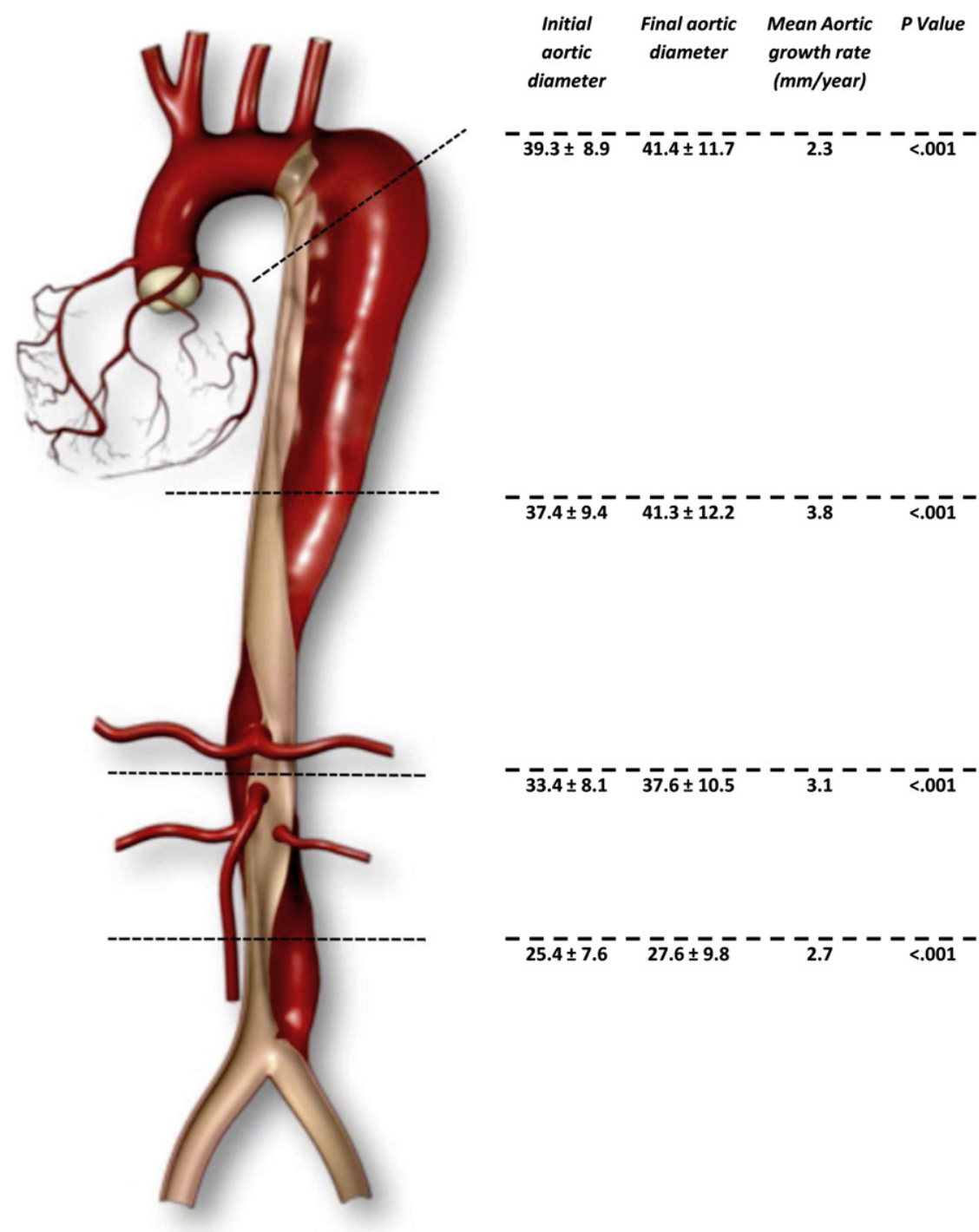

FIGURE 1. Aortic measurements and growth rates at the 4 levels.

hospital will die within 3 years. ${ }^{4,12}$ The blood flow dynamics of the false lumen in the subacute and chronic setting seem to be important prognostic predictors of patients with ABAD. Imaging studies suggest that complete thrombosis of the false lumen has beneficial prognostic value, whereas a patent false lumen predicts poor outcome. ${ }^{6-9}$ In a previous report from the IRAD investigators, the risk of death during follow-up in patients

TABLE 1. Aortic growth rates stratified according to false lumen status

\begin{tabular}{lcclr}
\hline & \multicolumn{1}{c}{ Partial } & \multicolumn{1}{c}{ Complete } & \\
& Patent & \multicolumn{1}{c}{ thrombosis } & thrombosis & $\boldsymbol{P}$ value \\
\hline Initial aortic diameter & $32.0 \pm 6.9$ & $38.0 \pm 12.0$ & $35.2 \pm 8.4$ & $<.001$ \\
Final aortic diameter & $34.7 \pm 7.7$ & $43.1 \pm 14.4$ & $39.3 \pm 12.2$ & $<.001$ \\
Mean aortic growth & $2.10 \pm 5.56$ & $4.25 \pm 10.18$ & $1.51 \pm 5.65$ & .069 \\
\hline
\end{tabular}

with a partial false lumen thrombosis was increased by a factor of 2.7 in comparison with patients with a completely patent false lumen. ${ }^{11}$ Because of the observational characteristics of the IRAD registry, it was unclear whether the higher mortality in patients with partial false lumen thrombosis was aorta related. The possible elevated pressure in the false lumen could result in increased aortic expansion and eventually rupture. ${ }^{11}$

The aim of the present study was to validate the relationship between partial false lumen thrombosis on computed tomography or magnetic resonance imaging and aortic expansion on follow-up imaging. In our analysis, we observed that the aortic growth rate was significantly increased among the aortic segments with a partially thrombosed false lumen compared with those with a patent false lumen. However, Sueyoshi and colleagues ${ }^{13}$ showed different findings. 
TABLE 2. Univariate linear regression analysis

\begin{tabular}{lcccc}
\hline & & \multicolumn{2}{c}{$\mathbf{9 5 \%} \mathbf{C I}$} & \\
\cline { 3 - 4 } \multicolumn{1}{c}{ Variable } & $\begin{array}{c}\text { Adjusted estimated } \\
\text { difference }(\mathbf{m m} / \mathbf{y})\end{array}$ & $\begin{array}{c}\text { Lower } \\
\text { bound }\end{array}$ & $\begin{array}{r}\text { Upper } \\
\text { bound }\end{array}$ & $\begin{array}{c}\boldsymbol{P} \\
\text { value }\end{array}$ \\
\hline Age at presentation & -0.015 & -0.23 & -0.06 & .001 \\
Male gender & 2.01 & 0.10 & 4.05 & .039 \\
Initial aorta diameter & 0.06 & -0.0 & 0.16 & .196 \\
$\begin{array}{l}\text { Patent (vs complete } \\
\quad \text { thrombosis) }\end{array}$ & 0.59 & -3.00 & 4.18 & .745 \\
$\begin{array}{l}\text { Partial thrombosis } \\
\quad \text { (vs Complete }\end{array}$ & 2.75 & -0.84 & 6.33 & .133 \\
$\quad$ thrombosis) & & & & \\
Partial thrombosis & & & & \\
$\quad$ (vs patent) & 2.15 & 0.16 & 4.15 & .035 \\
\hline$C I$, Confidence interval. & & & & \\
\hline
\end{tabular}

In their analysis, the dissected aorta was associated with the most rapid mean growth rate in case of a patent false lumen ( $4.9 \mathrm{~mm} /$ year), followed by a partially thrombosed false lumen $(4.0 \mathrm{~mm} /$ year $)$, whereas dissected aortas with a completely thrombosed false lumen decreased in diameter on average $(-0.2 \mathrm{~mm} /$ year). In the partially thrombosed false lumen group, dissected aortas with a "sac formation type" showed a significantly increased growth rate compared with those with a non-sac formation type. ${ }^{13}$ The differences between study groups are reflected by the fact that our study population had a higher percentage of partial lumen thrombosis compared with the study by Sueyoshi and collagues ${ }^{13}(47.6 \%$ vs $28.2 \%)$. These differences are possibly related to different scan protocols, different use of anticoagulation, and genetic, dietary, or environmental influences, although this remains highly speculative.

Our study revealed that patients with a partially thrombosed false lumen have a larger aortic diameter at presentation than other patients with dissection. At the different levels, the average diameter of dissected segments was larger in patients with partially thrombosed false lumens than in patients with a completely patent or a completely thrombosed false lumen. Multivariate analysis showed that the initial diameter did not have a significant effect on aortic growth, but it is possible that patients with a larger initial diameter might be more prone to develop

TABLE 3. Multivariate linear regression analysis

\begin{tabular}{lcrrr}
\hline & & \multicolumn{2}{c}{$\mathbf{9 5 \%}$ CI } & \\
\cline { 3 - 4 } \multicolumn{1}{c}{ Variable } & $\begin{array}{c}\text { Adjusted estimated } \\
\text { difference }(\mathbf{m m} / \mathbf{y})\end{array}$ & $\begin{array}{c}\text { Lower } \\
\text { bound }\end{array}$ & $\begin{array}{c}\text { Upper } \\
\text { bound }\end{array}$ & $\begin{array}{c}\boldsymbol{P} \\
\text { value }\end{array}$ \\
\hline Age at presentation & -0.17 & -0.26 & -0.08 & $<.001$ \\
Male gender & 2.30 & 0.32 & 4.28 & .023 \\
Partial thrombosis & 2.05 & 0.10 & 4.01 & .040 \\
$\quad$ (vs patent) & & & & \\
Initial aorta diameter & 0.05 & -.05 & .15 & .373 \\
\hline
\end{tabular}

CI, Confidence interval. thrombosis in the false lumen. Although an explanation for this association remains unclear at this moment, increased descending diameter in ABAD may be associated with preexisting aortic dilatation and atherosclerosis. ${ }^{13}$ These conditions may favor blood flow turbulences and thrombosis. We did not take into account the morphology of the aneurysm sac, so the association between partially thrombosed false lumen and aortic morphology needs to be more precisely defined.

Our analysis evidenced an equivalent incidence of partial thrombosis and total patency of the false lumen after dissection occurred, with a status of the false lumen that did not change during follow-up in more than $80 \%$ of aortic segments. However, aortic growth was more frequently observed among segments with a patent false lumen compared with those with partial thrombosis $(81.6 \%$ vs $78.4 \%$ ). Although this difference was not significant, it confirms that patency of the false lumen is a common anatomic condition after acute dissection and can play a primary role in determining descending aortic dilatation in the chronic setting. Patency of the false lumen has been reported as a predictor of aortic reoperation in the long-term of patients with type A dissection treated with ascending/arch open repair. ${ }^{14,15}$

This study showed increased aortic expansion in cases with a partially thrombosed false lumen. On the basis of these observations, these patients may benefit from a shorter surveillance interval or lower thresholds for prophylactic aortic intervention.

\section{Study Limitations}

The findings of the present study should be viewed within the light of its limitations. We did not include other morphologic characteristics of the aortic dissection, which may have affected aortic growth, except for the status of false lumen. Also, clinical data regarding baseline comorbidities and medical treatment were not available for analysis.

\section{CONCLUSIONS}

The aortic growth rate among patients with $\mathrm{ABAD}$ with a partially thrombosed false lumen seemed to be higher during follow-up than in patients with complete thrombosis or a patent false lumen. Therefore, patients with partial thrombosis may require more intensive follow-up and benefit from lower thresholds for prophylactic aortic intervention.

\section{References}

1. Elefteriades JA, Hartleroad J, Gusberg RJ, et al. Long-term experience with descending aortic dissection: the complication-specific approach. Ann Thorac Surg. 1992;53:11-20

2. Elefteriades JA, Lovoulos CJ, Coady MA, et al. Management of descending aortic dissection. Ann Thorac Surg. 1999;67:2002-5.

3. Suzuki T, Mehta RH, Ince H, et al. Clinical profiles and outcomes of acute type B aortic dissection in the current era: lessons from the International Registry of Aortic Dissection (IRAD). Circulation. 2003;108(Suppl 1):II312-7. 
4. Tsai TT, Fattori R, Trimarchi S, et al. Long-term survival in patients presenting with type B acute aortic dissection: insights from the International Registry of Acute Aortic Dissection. Circulation. 2006;114:2226-31.

5. Bernard Y, Zimmermann $\mathrm{H}$, Chocron S, et al. False lumen patency as a predictor of late outcome in aortic dissection. Am J Cardiol. 2001;87:1378-82.

6. Erbel R, Oelert H, Meyer J, et al. Effect of medical and surgical therapy on aortic dissection evaluated by transesophageal echocardiography. Implications for prognosis and therapy. The European Cooperative Study Group on Echocardiography. Circulation. 1993;87:1604-15.

7. Golledge J, Eagle KA. Acute aortic dissection. Lancet. 2008;372:55-66.

8. Mohr-Kahaly S, Erbel R, Rennollet H, et al. Ambulatory follow-up of aortic dissection by transesophageal two-dimensional and color-coded Doppler echocardiography. Circulation. 1989;80:24-33.

9. Sueyoshi E, Sakamoto I, Hayashi K, et al. Growth rate of aortic diameter in patients with type B aortic dissection during the chronic phase. Circulation. 2004;110:II256-61.
10. Trimarchi S, Nienaber CA, Rampoldi V, et al. Role and results of surgery in acute type B aortic dissection: insights from the International Registry of Acute Aortic Dissection (IRAD). Circulation. 2006;114:1357-64.

11. Tsai TT, Evangelista A, Nienaber CA, et al. Partial thrombosis of the false lumen in patients with acute type B aortic dissection. N Engl J Med. 2007;357:349-59.

12. Tsai TT, Schlicht MS, Khanafer K, et al. Tear size and location impacts false lumen pressure in an ex vivo model of chronic type B aortic dissection. $J$ Vasc Surg. 2008;47:844-51.

13. Sueyoshi E, Sakamoto I, Uetani M. Growth rate of affected aorta in patients with type B partially closed aortic dissection. Ann Thorac Surg. 2009;88: 1251-7.

14. Fattouch K, Sampognaro R, Navarra E, et al. Long-term results after repair of type a acute aortic dissection according to false lumen patency. Ann Thorac Surg. 2009;88:1244-50.

15. Park KH, Lim C, Choi JH, et al. Midterm change of descending aortic false lumen after repair of acute type I dissection. Ann Thorac Surg. 2009;87:103-8. 